\title{
Liver metastasis in colorectal cancer: evaluation of segmental distribution
}

\author{
Cengiz Kadiyoran ${ }^{1}$, Hilal Akay Cizmecioglu², Erkan Cure ${ }^{3}$, Mehmet Aykut Yildirim ${ }^{4}$, Pinar Diydem Yilmaz ${ }^{1}$ \\ ${ }^{1}$ Department of Radiology, Meram Medical Faculty, Necmettin Erbakan University, Konya, Turkey \\ 2Department of Internal Medicine, Meram Medical Faculty, Necmettin Erbakan University, Konya, Turkey \\ ${ }^{3}$ Department of Internal Medicine, Ota and Jinemed Hospital, Istanbul, Turkey \\ ${ }^{4}$ Department of General Surgery, Meram Medical Faculty, Necmettin Erbakan University, Konya, Turkey
}

Gastroenterology Rev 2019; 14 (3): 188-192

DOI: https://doi.org/10.5114/pg.2019.88168

Key words: liver, metastasis, colorectal carcinoma, Couinaud classification.

Address for correspondence: Ass. Prof. Cengiz Kadiyoran, Department of Radiology, Meram Medical Faculty, Necmettin Erbakan University, Beysehir St, 42080 Konya, Turkey, e-mail: ckadiyoran@hotmail.com

\begin{abstract}
Introduction: It is known that the liver is the main target for metastasis in colorectal cancer. However, we do not know enough from the literature to describe the segmental distribution of liver metastatic lesions of colorectal cancers.

Aim: To investigate which liver segment is affected.

Material and methods: A total of 326 patients (female/male, $n=115 / 221$; age: $53 \pm 8 / 51 \pm 7$ years) were included in our study, classified according to their pathological diagnosis. After liver metastases of the patients were determined, they were divided according to Couinaud classification.

Results: While the total number of metastases detected in the right lobe was 691 (70.1\%), the number of metastases detected in the left lobe was $294(29.9 \%)$, and the difference was highly significant $(p<0.0001)$. Metastases in the right lobe anterior segment amounted to 279 (40.4\%), and metastasis in right lobe posterior segment was 412 (59.6\%), and the difference was significant. When the total number of metastatic lesions is evaluated by excluding segment I, the largest number of lesions were observed in segment VIII. The liver segments with the highest number of metastatic lesions were, respectively, VII, IV, VI, V, III, and II. In this case, the fewest metastatic lesions were observed in segment II.

Conclusions: Liver metastases of colorectal cancer are more common in the right lobe than in the left lobe. The right lobe posterior segment (segment $\mathrm{VI}$ ) is the main target of metastases.
\end{abstract}

\section{Introduction}

Colorectal carcinoma (CRC) is a common type of cancer and is a common cause of cancer-related death [1]. Although the incidence and mortality rates have decreased recently, the reason for this decrease is not clear [1]. However, it is significant that the liver is the most likely target of metastasis in patients with CRC [2,3]. The correct diagnosis of metastatic disease is very important for the management of treatment. Early diagnosis prolongs surveillance of patients with CRC [4]. While the incidence of liver metastasis during the initial diagnosis of colon cancer is $14-18 \%$ but the incidence of liver metastasis is $10-25 \%$ when primary tumor surgery is performed. Radiological examinations will increase this rate by up to $35 \%$, and in $70 \%$ of patients with CRC liver metastasis will develop [5]. Although the treatment varies according to the number and size of the liver metastasis, it plays an important role in the localisation of the hepatic metastatic lesion. With these parameters, radiofrequency ablation (RFA), segmentectomy, lobectomy, transarterial-chemoembolisation (TACE), or chemotherapy treatment options will be used for liver metastases $[4,6]$. Radiological recognition, follow-up of liver metastases, and segment localisation of metastasis during surgical procedures are important $[7,8]$.
Aim
There are not enough studies in the literature about the distribution of liver metastases according to Cou- inaud classification [9], and there are even fewer patient populations in previous studies on the topic. The aim of our article is to provide an idea about the distribution of 
liver metastases by the method. Considering the large population included in our study compared to previous studies, we believe that it will contribute significantly to the literature about distribution of liver metastases.

\section{Material and methods}

\section{Study design and patients}

In our retrospective study, 326 patients with CRC were included, and the diagnoses of these patients were proven pathologically. Of the 326 patients, 196 had right-sided colon cancer (RSCRC) and 130 had left-sided colon cancer (LSCRC).

Three different methods were used in the diagnosis of liver metastases in these patients with known CRC. In the first, in a follow-up in a routine oncology outpatient clinic, the presence of a new lesion on abdominal computerised tomography (CT) was accepted as metastasis. Secondly, liver lesions were evaluated by Trucut biopsy in patients with known or newly diagnosed CRC. In the third method, patients were diagnosed with metastasis by radiological examination during the follow-up of the disease and pathologically proven metastasis after metastasectomy surgery.

Patients who had undergone liver surgery for any reason before they were diagnosed with CRC were excluded from the study. Caudate lobe (segment 1) lesions were excluded from the study because they are anatomically different from other lobes in that they often have direct connections to the inferior vena cava (IVC) through hepatic veins, which are separate from the main hepatic veins. Also, the caudate lobe may be supplied by both right and left branches of the portal vein. The caudate lobe (segment 1) frequently has direct linkages to the IVC through hepatic veins that are split from the main hepatic veins. Occasionally, both right and left branches of the portal vein may supply the caudate lobe. We excluded caudate lobe lesions from the study due to them being anatomically distinct from other lobes.

After detecting patients with liver metastasis, metastatic lesions for each patient were classified according to liver segments. In order to investigate the relationship between the side of colon cancer and right or left lobe metastasis of the liver, the metastatic segment was differentiated by the side of the CRC. Then, the results were evaluated.

\section{Statistical analysis}

The data obtained were evaluated statistically with the help of SAS University Edition 9.4 software. Generalised mixed model was used to compare data.

\section{Results}

A total of 326 patients were included in our study, of whom 115 were female and 221 were male. The mean age of the female patients was $53 \pm 8$ years, and the mean age of the male patients was $51 \pm 7$ years. The distribution and number of patients included in the study by primary pathology and gender are classified in Table I.

While the total number of metastases detected in the right lobe was 691 (70.1\%), the number of metastases detected in the left lobe was 294 (29.9\%), and the difference was statistically significant $(p<0.0001)$. The total metastatic lesion distribution to all segments is documented in Table II.

Metastases in the right lobe anterior segment numbered 279 (40.4\%), and metastases in right lobe posterior segment totalled 412 (59.6\%); the difference was statistically significant.

When the total number of metastatic lesions was evaluated by excluding segment I, the largest number of lesions were observed in segment VIII. The liver segments with the highest number of metastatic lesions were, respectively, VII, IV, VI, V, III, and II. In this case, the least metastatic lesions were observed in segment II. The results are summarised in Table III.

Patients with metastatic lesions only in the right hemiliver numbered 128 (39.2\%), 41 (12.6\%) patients had metastases only in the left hemiliver, and 157 (48.2\%) patients had metastatic lesions in both right and left hemilivers. Only 55 of these 326 patients had single segment metastases in the right hemiliver, and $27 \mathrm{pa}-$ tients had only single segment metastases in the left hemiliver.

Table I. Distribution and number of patients included in the study by primary tumour side and gender

\begin{tabular}{|c|c|c|c|c|c|}
\hline \multirow[t]{2}{*}{ Parameter } & \multicolumn{2}{|c|}{ Right-sided colon cancer $(n=117)$} & \multirow[t]{2}{*}{ Parameter } & \multicolumn{2}{|c|}{ Left-sided colon cancer $(n=209)$} \\
\hline & Male & Female & & Male & Female \\
\hline Caecum & 28 & 17 & Descending colon & 22 & 14 \\
\hline Ascending colon & 30 & 15 & Sigmoid colon & 54 & 25 \\
\hline Transverse colon* & 17 & 10 & Rectum & 60 & 34 \\
\hline
\end{tabular}

*Proximal 2/3 part of transverse colon. 
Table II. Liver metastases distribution of colonic tumour side for each patient

\begin{tabular}{lcccccc} 
& \multicolumn{3}{c}{ Female $(\boldsymbol{n}=\mathbf{1 1 5})$} & \multicolumn{3}{c}{ Male $(n=211)$} \\
\cline { 2 - 7 } & Right & Left & Left and right & Right & Left & Left and right \\
\hline Rectum & 11 & 2 & 21 & 31 & 6 & 23 \\
\hline Sigmoid colon & 10 & 4 & 11 & 17 & 8 & 29 \\
\hline Descending colon & 3 & 5 & 6 & 7 & 5 & 10 \\
\hline Transverse colon* & 4 & 2 & 4 & 13 & 3 & 10 \\
\hline Ascending colon & 6 & - & 9 & 12 & 3 & 14 \\
\hline Caecum & 7 & 3 & 7 & & 7 & 13
\end{tabular}

*Proximal 2/3 part of transverse colon.

Table III. Distribution of metastases obtained in the segments of the liver

\begin{tabular}{lcccccccc} 
Parameter & \multicolumn{9}{c}{ Left lobe } & \multicolumn{3}{c}{ Right lobe } \\
\hline Segment & II & III & IV & V & VI & VII & VIII \\
\hline Involved time & 113 & 95 & 86 & 157 & 234 & 178 & 122 \\
\hline$\%$ & 11.4 & 9.6 & 8.7 & 15.9 & 23.7 & 18 & 12.3
\end{tabular}

Fifty-five (16.3\%) patients with metastases underwent surgery, and pathological confirmation was achieved in these patients. In 111 (34\%) patients, pathology correlation was achieved with Tru-cut biopsy.

A metastatic lesion seen at the left lobe lateral segment on contrast enhanced axial CT (Figure $1 \mathrm{~A}$ ) and the same lesion seen at the PET (Figure $1 \mathrm{~B}$ ) imaging. And at the dynamic CT of the liver; a metastatic lesion (white arrow) seen at the right lobe anterior segment. Note the capsuler wash out at portal phase and late phase compared with arterial phase (Figures $2 \mathrm{~A}-\mathrm{C}$ ).

Metastatic liver lesions show independent distribution by primary cancer side. In our study men with

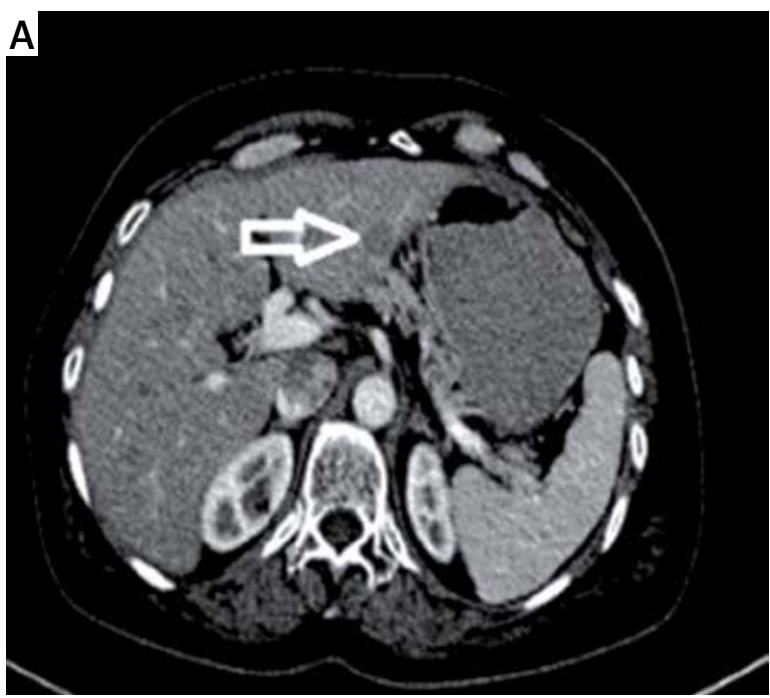

rectal cancer, the most common metastases detected is the right lobe. And at the female patients with rectal cancer right lobe and left lobe involvement is observed simultaneously. In both male and female patients with sigmoid colon cancer, the most common side of metastatic involvement is left and right lobe coexistence.

\section{Discussion}

Metastatic disease affects the right lobe of the liver more than the left lobe [3]. There are various theories about this. There are reports that the right lobe volume and weight of the right lobe of the liver are higher than

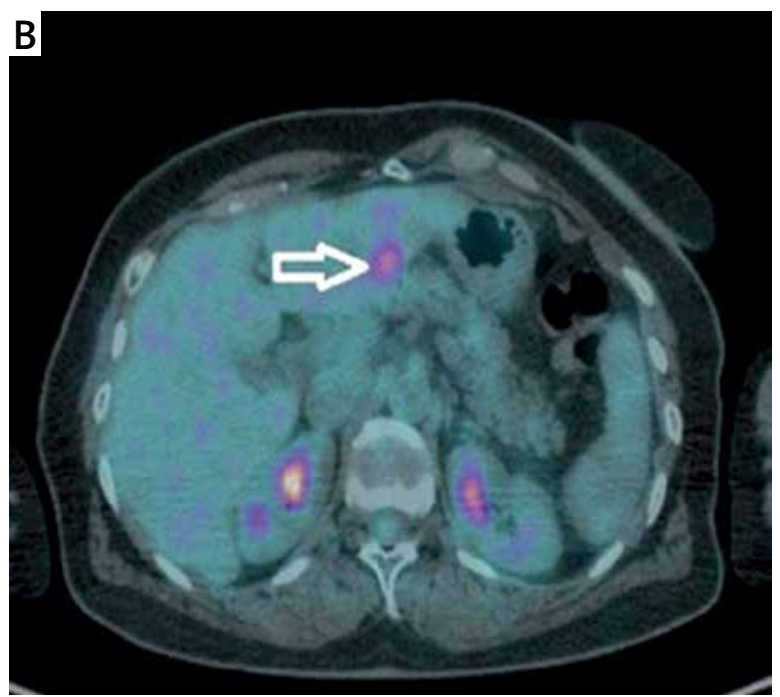

Figure 1. A metastatic lesion seen at the left lobe lateral segment on contrast enhanced axial CT (A) and PET (B) imaging 


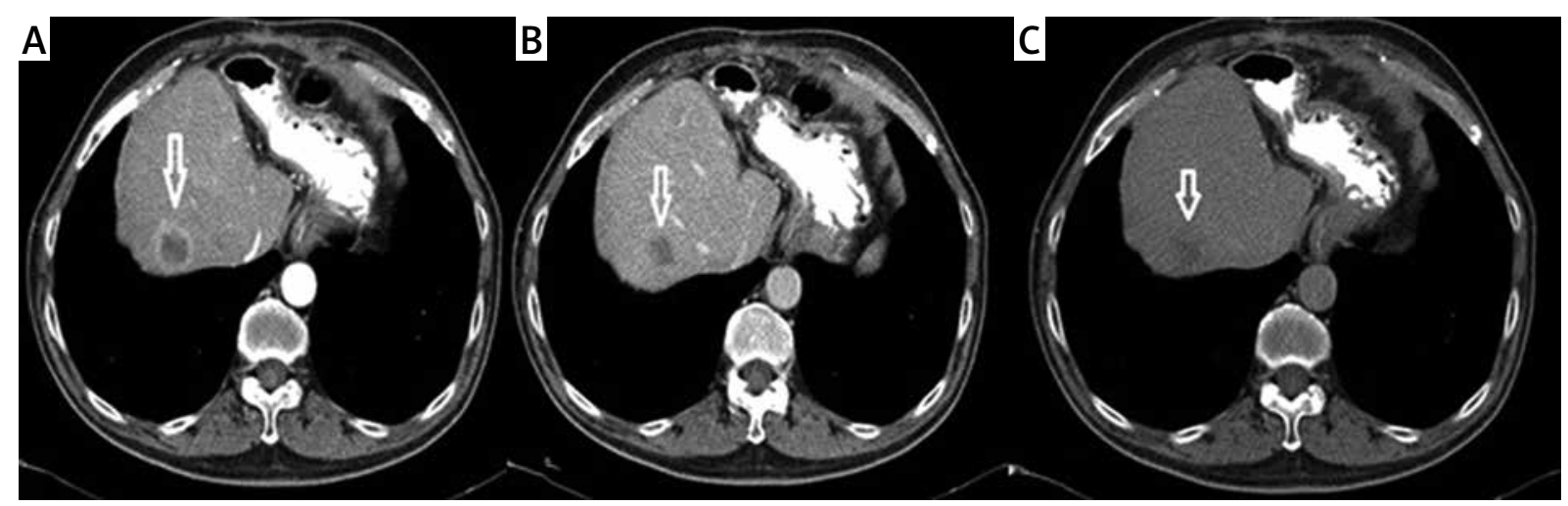

Figure 2. Metastatic lesion (white arrow) seen at the right lobe anterior segment. A - Arterial phase (capsular ring enhancement). B - Portal phase (capsular wash out). C - Late phase (capsular wash out)

those of the left lobe of the liver [3]. Thus, the number of metastatic lesions will be higher as the right lobe blood flow increases. $2 / 3$ of the liver blood flow is provided by the portal vein and especially the right lobe has highest portal vein flow and lowest hepatic artery flow [10].

In the study performed by Shirai et al., it was stated that right colon tumors were spread 10 times more than the left lobe of the liver compared to the right lobe and the left colon and rectum tumors were spread 2 times more in the right lobe compared to the left lobe [11]. Dionne examined hematogenous metastases of patients with rectal cancers and reported no difference in lobar distribution in the liver [12]. In our study, the presence of more metastases in the right lobe of the liver than in the left lobe was determined and correlated with the literature.

In addition, it has been reported that the liver blood flow is homogeneously distributed in relation to the segment of the liver and thus the volume of the liver lobe [3]. However, Schulz et al. [13] and Dionne [12] reported a heterogeneous distribution of liver portal vein flow. Lautt found that liver right lobe took the major part of the portal vein flow and as well as the least hepatic arterial flow in dogs and cats [14]. Many publications, report that the right lobe receives more blood flow from the portal vein than the left lobe due to both volume and weight differences, and this is attributed to the greater observation of the right lobe in metastatic lesions [2, $3,10,15]$. Wigmore et al. found 708 metastatic lesions in 207 patients with CRC [3]. In a study performed by Rhu et al., a total of 652 metastases were confirmed histopathologically; there were 398 metastases (61\%) found in the right lobe and 254 metastases (39\%) were detected in the left lobe. Finally total ratio of right to left lobe is $1.57: 1$ [15]. In our study, we determined 691 (70.1\%) metastases in the right lobe and 294 (29.9\%) metastases in the left lobe, which were found to be correlated with the studies of Rhu et al. and Wigmore et al.
It is clear that the portal vein plays a key role in the segmental distribution of liver metastases. The portal vein is one of the important points in the bifurcation level. The right portal vein is the continuation of the main portal vein while the left portal vein is separated from the main portal vein at an acute angle. This is the reason why the blood flow from the portal vein to the left lobe is less, while the continuity of the right portal vein directly with the main portal vein is another factor affecting the blood flow [16]. According to the portal variation classification described by Cheng et al. [17], this bifurcation constitutes $70.9-86.2 \%$ of total cases. This study explains the reasons why more metastases were detected in the right lobe. Interestingly, Shirai et al. suggested that right hepatectomy would remove $90 \%$ of the liver metastases from the liver in patients with right colon tumours [11]. Shirai et al. point to the right lobe as a target.

Although the literature states that the right lobe involvement is greater than the left in most of the publications, many publications have not reached a large enough series of patients to reveal this, and there are no guiding numerical values. Our study reached a wide range of patients: 985 metastatic lesions detected in 326 patients; $70.1 \%$ of the metastatic lesions were found in the right lobe and $29.9 \%$ in the left lobe. Another important point is to investigate the relationship between the side of CRC and right or left lobe metastasis of the liver. When the RSCRC and LSCRC and the side of metastasis in the liver is investigated; it is clearly understood that the liver metastasis are seen more common at the right lobe than the left lobe of the liver. However, it was not clear that RSCRC were more metastase to liver right lobe and LSCRC were more metastase to left lobe of the liver. In our study, we could not detect a correlation between the side of the CRC and the side of the liver metastasis. 


\section{Conclusions}

The liver is an important target for CRC. Our study reached a greater patient population than many studies in the literature, and according to our study, liver metastases of CRC are more common in the right lobe than in the left lobe. The right lobe posterior segment (segment VI) is the main target of metastases. Liver metastasis from the right or left colon tumours is not a descriptive finding.

\section{Conflict of interest}

The authors declare no conflict of interest.

\section{References}

1. Ansa BE, Coughlin SS, Alema-Mensah E, et al. Evaluation of colorectal cancer incidence trends in the United States (20002014). J Clin Med 2018; 7: 22.

2. Strohmeyer T, Schultz W. The distribution of metastases of different primary tumors in the liver. Liver 1986; 6: 184-7.

3. Wigmore SJ, Madhavan K, Redhead DN, et al. Distribution of colorectal liver metastases in patients referred for hepatic resection. Cancer 2000; 89: 285-7.

4. Sica GT, Ji H, Ross PR. CT and MR imaging of hepatic metastases. Am J Roentgenol 2000; 174: 691-8.

5. Valderrama-Trevino Al, Barrera-Mera B, Ceballos-Villalva JC, et al. Hepatic metastasis from colorectal cancer. Euroasian J Hepatogastroenterol 2017; 7: 166-75.

6. Lee HY, Chung JW, Lee JM, et al. A new and simple practical plane dividing hepatic segment 2 and 3 of the liver: evaluation of its validity. Korean J Radiol 2007; 8: 302-10.

7. Engstrand J, Nilsson N, Strömberg C, et al. Colorectal cancer liver metastases - a population-based study on incidence, management and survival. BMC Cancer 2018; 18: 78.

8. Kadiyoran C, Cizmecioglu HA, Cizmecioglu A, et al. Comparison of the gadoxetic acid and gadopentate dimeglumine efficiency for determining liver metastases by an enhanced MRI of patients with gastrointestinal malignancies. Selcuk Med J 2018; 34: 148-54.

9. Couinaud C. Le foie: etudes anatomiques et chimgicales. Masson and Cie, Paris: 1957; 3.

10. Holbrook RF, Rodriguez-Bigas MA, Ramakrishnan K, et al. Patterns of colorectal liver metastases according to Couinaud's segments. Dis Colon Rectum 1995; 38: 245-8.

11. Shirai Y, Wakai T, Ohtani T, et al. Colorectal carcinoma metastases to the liver: does primary tumor location affect its lobar distribution? Cancer 1996; 77: 2213-6.

12. Dionne $L$. The pattern of blood borne metastasis from carcinoma of the rectum. Cancer 1965; 18: 775-81.

13. Schulz W, Hagen C, Hort W. The distribution of liver metastases from colonic cancer: a quantitative postmortem study. Virchows Arch Pathol Anat Histopathol 1985; 4056: 279-84.

14. Lautt WW. Hepatic Circulation: Physiology and Pathophysiology. Morgan \& Claypool Life Sciences, San Rafael (CA) 2009.

15. Rhu J, Heo JS, Choi HS, et al. Streamline flow of the portal vein affects the lobar distribution of colorectal liver metastases and has a clinical impact on survival. Ann Surg Treat Res 2017; 92: 348-54.
16. Lencioni R, Cioni D, Bartolozzi C. Focal Liver Lesions. Detection, Characterization, Ablation. Springer 2005.

17. Cheng Y, Huang T, Lee T, et al. Variation of intrahepatic portal vein; angiographic demonstration and application in living related hepatic transplantation. Transplant Proc 1996; 28: 1667-8.

Received: 1.01.2019

Accepted: 17.01.2019 